\title{
La Polynésie française à la recherche de son identité
}

\section{Gilles Blanchet}

\section{(2) OpenEdition \\ 1 Journals}

Édition électronique

URL : http://journals.openedition.org/jso/1127

DOI : $10.4000 /$ jso. 1127

ISSN : $1760-7256$

\section{Éditeur}

Société des océanistes

\section{Édition imprimée}

Date de publication : 1 juin 2003

Pagination : $3-12$

ISSN : 0300-953x

\section{Référence électronique}




\section{La Polynésie française à la recherche de son identité}

par

Gilles BLANCHET *

\section{RÉSUMÉ}

Cet article s'interroge sur la montée des revendications d'identité et des recompositions identitaires en Polynésie française, un territoire d'outre-mer qui vient de connaître des turbulences et semble aujourd'hui à la croisée des chemins avec un amoindrissement de l'emprise de l'État et un renforcement du pouvoir des autorités territoriales. La suspension des expériences nucléaires en 1992 et leur arrêt définitif en 1996 ont donné lieu à une série de négociations entre les autorités territoriales et nationales en vue d'atténuer les conséquences d'une mesure qui prive le territoire de sa principale source de revenus. Elles ont tour à tour débouché sur une loi d'orientation, un Pacte de Progrès, un statut d'autonomie renforcée et un Programme stratégique aux termes desquels l'État s'engage à verser au Territoire des compensations financières pendant dix ans. Tous ces changements ont transformé les rapports entre la métropole et le Territoire et modifié les relations entre Tahiti et les autres îles. Ils ont parallèlement engendré au sein de la population une crise d'identité et une recherche de racines qui se poursuivent aujourd'hui. C'est cette évolution qu'aborde cet article qui considère la notion d'identité moins comme une donnée et un substrat culturel primordial que comme le fruit provisoire de stratégies conduites par des agents déterminés.

MotS-CLÉS: Essais nucléaires, identité, autonomie, dépendance, rapports entre Tahiti et la métropole, rapports entre Tahiti et les archipels éloignés

\section{ABSTRACT}

This paper looks at identity problems in French Polynesia, an overseas territory confronted with crucial changes after French government's decision to stop nuclear testing in Tuamotu archipelago. Nuclear experiments which started in the mid-sixties have ended for good in 1996 after a three years' moratorium and an ultimate range of tests. Then, to prepare the after-CEP period, national and territorial authorities launched negotiations which lead onto an orientation law and a strategic program attesting French commitment to the territory and ensuring him important financial compensations for the next ten years. As for French Polynesia authorities, they engaged themselves to allocate the money to productive activities in order to reduce dependency from the mother country. Concomitantly, a new status was elaborated giving greater autonomy to the Territory and enabling him to develop relations with other countries on the regional and international scene. All these changes associated with difficult times have locally generated an identiy crisis and a search for roots which is still going on. They have also opened onto a new pattern of relationship between the Territory and the mother country as well as between Tahiti and outer islands. The trend is particularly strong in remote islands which have been durably kept out of development policies and financial support all along the past years. This paper is committed to these topics and looks at identity not as a fixed cultural feature but as a complex and evolutive process set up by well identified agents.

KeywORDS : Nuclear experiments, identity, dependency, relations between Tahiti et French government, relations between Tahiti and remote islands.

\footnotetext{
* Économiste à l'IRD.
} 
La Polynésie française à la recherche de son identité

Depuis quelques années, la multiplication des revendications d'identité et des recompositions identitaires en Polynésie française amène à s'interroger sur leur signification profonde. Un regard en arrière suggère que la revendication d'une identité propre n'est pas nouvelle mais se produit de manière récurrente dans l'histoire du territoire depuis sa découverte par les Occidentaux. À la différence de ce qui a pu se passer dans d'autres pays colonisés, la singularité des Polynésiens a pourtant été immédiatement reconnue par les explorateurs et les voyageurs comme par les missionnaires et les colonisateurs, même si elle a été perçue de l'extérieur et souvent élevée au rang du mythe.

Quant aux insulaires, leurs revendications identitaires se sont d'abord manifestées à une échelle locale pour des raisons qui tiennent aux trajectoires qu'ils ont suivies, à leurs modes de vie et au jeu des forces en présence. Les habitants des archipels des Australes, des Marquises et des Tuamotu-Gambier, par exemple, se sont toujours appliqués à souligner ce qui les différencie les uns des autres et les distingue de Tahiti et de Papeete, la capitale et chef-lieu du Territoire. Cela ne les a pas empêchés, dans le contexte de la décolonisation, de se réclamer de leur qualité de français d'origine polynésienne chaque fois qu'ils ont eu à se prononcer sur le sujet lors de référendums ou autres échéances électorales.

Ils ont pareillement adhéré au sentiment d'identité qui a pris forme au niveau régional et été synthétisé sur le plan politique par la 'Pacific Way', une notion commune à l'ensemble des territoires insulaires du Pacifique Sud, mettant l'accent sur des relations basées sur le dialogue et le consensus. Le concept s'est répandu comme une traînée de poudre au milieu des années 70 et a été épaulé sur le plan culturel par la référence à une même origine et une même culture maohi. Au début des années 80 , il devient un véritable leitmotiv et, à côté de la 'Polynesian Way' d'abord prévalente, se développe une 'Melanesian Way' qui met davantage l'accent sur la coutume, un mode de vie communautaire et des relations plus égalitaires.

Au cours des années 90 , ces thèmes perdent de leur force mobilisatrice en raison même de leur succès et du hiatus croissant qui se fait jour entre le niveau abstrait où sont posés les problèmes et le niveau concret où il faut les résoudre.

Avec les progrès des moyens de communication, l'accélération du processus de mondialisa- tion, le développement des interdépendances et la montée du libéralisme, les revendications d'identité se transforment elles aussi. Elles se posent moins désormais sur le plan national que sur les plans infra et supra national et moins sur le terrain de l'idéologie et des grands principes que sur celui, plus prosaïque, des intérêts bien compris

Une telle évolution est difficile à imaginer au début des années 1960 lorsque la Polynésie française voit sa situation brusquement changer avec le choix de deux îles des Tuamotu comme lieux d'expériences nucléaires et l'implantation à Tahiti du Centre d'Expérimentation du Pacifique (CEP). La vie locale en est transformée et c'est dans le sillage du CEP que va de plus en plus se couler l'économie du Territoire jusqu'à ce que, trente ans plus tard, sa fermeture vienne à nouveau modifier la donne.

La suspension des essais de 1992 à 1995 et leur arrêt définitif en 1996 soulèvent une légitime inquiétude sur l'avenir et donnent lieu à toute une série de discussions et de pourparlers entre les autorités locales et nationales. Elles débouchent, dans un premier temps, sur une loi d'orientation et un Pacte de Progrès et, dans un second temps, sur un statut d'autonomie renforcée et un programme stratégique aux termes desquels l'État s'engage à verser un milliard de francs (15,2 millions d'euros) chaque année au territoire pendant dix ans, à titre de compensation. Celui-ci s'engage, de son côté, à consacrer ces sommes au développement de ses capacités productives de manière à réduire sa dépendance de la métropole.

L'importance et le caractère radical de ces changements, comme la longue période d'incertitude et de crise qui les précède, ont un effet traumatisant sur la population et entraînent une crise d'identité et une recherche de racines qui se poursuivent aujourd'hui. Ils conduisent parallèlement à une redéfinition des relations du Territoire avec la France et à une recomposition des rapports existant entre Tahiti, au cœur des îles de la Société, et les autres îles. Cette évolution est particulièrement nette dans les archipels éloignés qui sont longtemps restés à l'écart des politiques de développement mises en œuvre et ont moins bénéficié que d'autres de la manne venue de l'extérieur.

Dans un contexte aussi volatil, l'identité apparaît moins comme une donnée et un substrat culturel primordial que comme une construction provisoire aux contenus parfois contradictoires. Elle n'en est pas moins au cœur des stratégies impulsées par les dirigeants politiques et responsables administratifs qui sont eux-mêmes 
confrontés à des situations complexes dont il est malaisé de mesurer les tenants et aboutissants.

Cela tient pour une part à l'ambiguïté même de la notion d'identité qui apparaît à la fois comme un facteur d'union et de séparation et importe autant par ce qu'elle met en lumière que par ce qu'elle élude. Ce n'est pas un hasard si on y recourt surtout dans des circonstances difficiles où il faut resserrer les rangs pour retrouver une unité perdue, définir une ligne de conduite homogène et consolider l'autorité en place.

Ce semble être le cas de la Polynésie française et c'est ce qu'aborde cet exposé. Dans une première partie, il se focalise sur le questionnement identitaire qui se produit au début des années 1990 dans une atmosphère de crise qui atteint son paroxysme avec l'arrêt des essais nucléaires. Dans une seconde partie, il se tourne vers les institutions et analyse l'évolution des rapports entre l'État et le Territoire qui se marque par un désengagement progressif de l'État et une hégémonie croissante de Tahiti sur les îles périphériques situées à des milliers de kilomètres de là.

\section{Une société en crise qui s'interroge sur son identité}

L'arrêt des essais nucléaires survient dans une conjoncture de récession économique et de chômage et dans une atmosphère de crise qu'émaillent des bouffées de violence comme la grève des dockers du port de Papeete en octobre 1987, de nouvelles grèves et le blocus routier de Papeete en 1991 et 1992 ou la prise d'assaut de l'aéroport de Faaa et le sac de la ville de Papeete en septembre 1995. À cela s'ajoutent une série d'affaires judiciaires où se mêlent la politique et la finance et un endettement croissant du territoire dont le déficit n'est couvert en 1990 que grâce à un emprunt contracté auprès d'une banque privée.

Le désengagement de l'État s'opère lui-même par à-coups, au rythme de la réduction des dépenses militaires et des échéances politiques. Le manque de programmation et les incertitudes qui accompagnent cette évolution ont un effet déstabilisant qui aggrave le climat de crise.

Le Comité économique, social et culturel (CESC) de Polynésie française ${ }^{1}$ résume assez bien la situation lorsqu'il écrit en 1992 qu'on assiste à une perte de confiance généralisée du Territoire envers l'État, de la population envers ses élus, des administrés envers la justice, des investisseurs envers la Polynésie et de la Polynésie envers ellemême. Ce désarroi se transforme en inquiétude lorsqu'est prise en avril 1992 la décision 'unilatérale et brutale' de suspendre les essais. La question de l'avenir du Territoire privé de la rente nucléaire qui lui fournit l'essentiel de ses ressources passe alors au premier plan et des pourparlers sont rapidement entamés avec Paris pour trouver une solution de rechange. Ils débouchent en mai 1992 sur un accord et la mise en place d'un Pacte de Progrès inspiré des Accords Matignon signés en 1988 en NouvelleCalédonie.

Il s'agit d'un véritable programme de développement susceptible d'accompagner la mutation du territoire et le protocole d'accord stipule qu'il « appartient aux autorités territoriales que le statut d'autonomie interne a doté de larges compétences de définir prioritairement les axes de développement économique, social et culturel de la Polynésie française ».

C'est alors que le CESC de Polynésie française, prenant appui sur le statut d'autonomie interne de 1984 (article 88), décide de s'auto-saisir et d'entamer une réflexion sur l'avenir de la Polynésie. L'initiative fait boule de neige et un effort général de réflexion et de concertation est entrepris dans le cadre d'une Charte de Développement qui doit servir de base pour l'élaboration du futur Pacte de Progrès. Y participent les forces vives du Territoire, politiques, économiques et culturelles aussi bien qu'associatives, administratives ou religieuses.

À l'avis du CESC viennent ainsi s'ajouter une Charte de l'Éducation, les commentaires de 32 ateliers de réflexion réunis sous forme d'états généraux du développement, les remarques des maires des communes des Marquises et des Tuamotu et les enseignements tirés d'une consultation générale des Églises, syndicats et associations.

Ces propos sont rassemblés dans un document de synthèse que présente à Paris une délégation polynésienne de la Charte du Développement composée des présidents du gouvernement, de l'Assemblée territoriale, du CESC ainsi que des parlementaires polynésiens. Un autre document de synthèse est simultanément élaboré par les services de l'État. Ces deux

1. Le CESC est un organe consultatif qui formule des avis dans les domaines les plus divers à la demande du gouvernement polynésien ou de l'Assemblée territoriale. Il est composé de représentants des groupements professionnels, des syndicats, des organismes et des associations qui concourent à la vie économique sociale et culturelle de la Polynésie française. La loi organique du 12 avril 1996 limite ses possibilités d'auto-saisine et ne l'autorise plus à réaliser des études sur des thèmes inscrits à l'ordre du jour de l'Assemblée territoriale (article 90). 
rapports servent de référence dans les négociations entre l'État et le Territoire qui débouchent sur un Pacte de Progrès en janvier 1993 et sur une loi d'orientation au début de l'année suivante.

Au cours d'une période aussi brève qu'exceptionnelle, où chacun a pu s'exprimer librement, se sont ainsi fait entendre des points de vue très divers, éloignés des sentiers battus et aux antipodes de la langue de bois et des discours convenus.

On ne retiendra ici que ceux qui ont trait à l'identité du Territoire et à celle de ses habitants.

\section{Le constat d'une identité polynésienne en péril}

Le constat le plus souvent formulé est celui d'une société en crise qui assiste, impuissante, à la disparition rapide de ce qui était traditionnellement constitutif de son identité.

Le document de travail remis au ministre des DOM-TOM par la Délégation polynésienne de la Charte de Développement en juillet 1992 déplore la difficulté croissante du Territoire à associer valeurs polynésiennes et valeurs occidentales. Il constate que « le mode de vie actuel déstructure le cadre familial traditionnel désormais éclaté dans l'espace et le temps en de multiples lieux d'activité. Il détruit les valeurs communautaires fondamentales de solidarité et de convivialité, de respect des êtres. Il contribue à promouvoir un individualisme forcené dans lequel le Polynésien ne se reconnaît plus [...]. C'est ainsi qu'on assiste à la dislocation de la structure familiale, à la perte d'autorité parentale à l'absence de statut de l'enfance et de la jeunesse et la perte des références culturelles que vient aggraver le développement des sectes $»$.

Le même document insiste sur le fait qu'on assiste à la disparition des valeurs traditionnelles et non à leur transformation ou à l'émergence de valeurs nouvelles susceptibles de rallier et unifier les cultures des différentes ethnies présentes dans le Territoire. Plus inquiétant encore, "sous la pression incontrôlée des modèles extérieurs et au détriment des valeurs polynésiennes, une nouvelle culture difforme de type urbain se met en place, favorise l'individualisme au détriment de la communauté et pratique l'hégémonie sur les cultures des autres archipels ».

La Polynésie française serait donc en train de perdre ses valeurs coutumières et n'arriverait pas à en élaborer de nouvelles. En serait responsable le système économique mis en place par l'État depuis 1963 qui est venu bouleverser les bases traditionnelles de l'économie et l'a rendue dépendante des transferts publics. Cette situation a favorisé le développement des clivages sociaux et débouché sur une société à deux vitesses et sur l'émergence d'un quart monde de laissés-pour-compte qui représente un risque permanent d'explosion sociale.

Le dilemme est que les autorités en place semblent incapables de faire face à la situation et ne réussissent pas à s'imposer face aux forces en présence qui défendent le 'statu quo ante' et freinent toute évolution contraire à leurs opinions et leurs intérêts.

Le rapport du CESC explique ce blocage par l'existence de quatre grands groupes ethniques et de quatre grandes couches sociales qu'il identifie ainsi :

À la base, une « communauté maohi (59\% de la population au recensement de 1988), attirée par l'occidentalisation et le progrès économique, s'écarte peu à peu de sa culture d'origine sans pour autant trouver une nouvelle identité culturelle, d'où le sentiment de frustration qui l'accompagne, sentiment exacerbé par la condition de prolétariat dans laquelle elle est cantonnée. Elle tend ainsi à constituer une classe sociale dominée économiquement et de plus en plus touchée par le chômage ».

$\mathrm{Au}$ sommet, une couche popaa (19\% de la population), composée de français de passage qui vivent en circuit fermé.

S'y ajoutent deux autres couches sociales :

Une couche moyenne d'origine chinoise (6,5\% de la population), bien intégrée sur le plan économique à travers des circuits occidentaux et non polynésiens, qui souhaiterait avoir accès aux rouages politiques.

Une couche «demie » $(15,5 \%$ de la population), qui s'apparente à une classe dirigeante et se montre soucieuse de conserver son pouvoir et ses privilèges. Elle bénéficie du double apport des cultures européenne et polynésienne et se distingue à la fois du monde occidental et du monde maohi dont elle est issue.

Tous ces clivages expliquent l'incapacité de la classe politique à maîtriser une situation qui ne cesse de se dégrader avec une forte croissance démographique, une diminution des fonds en provenance de la métropole et une conjoncture économique morose. Simultanément, le chômage s'accroît avec l'arrivée chaque année de 3000 jeunes sur le marché du travail. Les phénomènes d'exclusion se développent et toucheraient $20 \%$ de la population, ce qui est d'autant plus inquiétant que la solidarité familiale, qui caractérisait la société polynésienne traditionnelle, ne joue plus en zone urbaine (Lanier et Allouche, 1996). 
En découlent un climat grandissant de contestation et des « poussées de fièvre » et « levées de boucliers » contre la reprise des essais nucléaires ou contre des investissements immobiliers et miniers qui bousculent les usages et font fi de la très forte relation à la terre des personnes concernées.

\section{L'appel à une identité au carrefour des cultures}

Pour remédier à tout cela, il faudrait arriver à concilier une organisation culturelle traditionnelle, qui repose sur les notions de partage, de solidarité et de respect de la parole donnée, et une société moderne centrée sur le marché qui confère une valeur marchande à ce qui était considéré jusque là comme faisant partie du patrimoine culturel.

Pour la Charte de Développement, la Polynésie française ne peut faire l'impasse ni sur ses racines culturelles auxquelles elle reste attachée ni sur une modernité dont elle ne saurait plus se passer. Elle doit, en conséquence, avoir pour objectif «de contribuer à forger une nouvelle identité culturelle qui constitue le ciment de la société polynésienne contemporaine plurielle et pluriethnique. Cette nouvelle identité doit puiser ses sources à la fois dans ses traditions et la modernité ainsi que dans les différentes cultures qui se sont mélangées dans le Territoire. Elle doit avoir pour ambition la richesse permise par l'apport combiné des valeurs polynésiennes, occidentales et asiatiques $»$.

Cela suppose le respect des valeurs qui se rattachent à la famille, à l'accueil d'autrui et au respect de la terre, éléments clés de la culture polynésienne. Cela implique aussi une ouverture sur des valeurs universelles telles que le respect des droits de l'homme et du citoyen ou le refus du racisme et de tout ostracisme. "C'est dans ces conditions que, réconciliés avec leur culture et rassurés dans leur identité, les hommes et les femmes de Polynésie, en puisant dans leurs racines, sauront intégrer tous les apports de leur histoire et s'ouvrir à une vision dynamique de leur devenir dans une volonté d'épanouissement $»$.

C'est aussi la position du CESC dont le rapport sur l'avenir du Territoire commence par affirmer que «La Polynésie doit, malgré une situation difficile et devant un avenir incertain, se reprendre pour faire face à l'adversité du moment, se déterminer sur un modèle de société qui lui semble le plus compatible avec ses aspirations profondes, repenser son mode de développement et son espace de vie, réfléchir enfin sur son cadre institutionnel ».
Il constate que l'absence d'évolution de l'ancien système et l'adoption d'un modèle calqué sur la métropole se juxtaposent avec un brassage ethnique et une ouverture sur le monde extérieur qui entraînent une acculturation de la majorité de la population au monde moderne. Il faut donc, pour que la société polynésienne retrouve sa cohésion et foi en l'avenir qu'elle conjugue la modernité et sa culture propre, ce que pourrait faciliter le mouvement de retour aux sources et de renouveau culturel qui se développe depuis la fin des années 70 .

Esquissant les assises sur lesquelles pourrait reposer la société de demain, le CESC la voit, lui aussi, comme une société pluriethnique et pluriculturelle fondée sur les droits de l'homme et les valeurs liées à son appartenance à la chrétienté, adhérant à la démocratie aussi bien qu'à l'État de droit. La société actuelle 'néo-polynésienne' lui apparaît finalement comme une "société maohi qui s'est enrichie au contact d'autres cultures pour former une nouvelle identité culturelle commune à tous les habitants du pays, quelle que soit leur origine ethnique. La société polynésienne est désireuse de démocratie, de justice et de solidarité. Pluriethnique et multiculturelle par nécessité historique et future, elle doit être à l'écoute de l'autre et ouverte au monde. Cette Nouvelle Société Polynésienne ne doit pas être un simple pari culturel mais doit se fonder sur un véritable programme politique et sur la prévision et la planification d'un avenir pluriculturel $»$.

Pour y parvenir, il serait souhaitable de faire une pause et de renforcer les acquis avant de poursuivre un développement qui a été trop rapide pour être maîtrisé. Il importe aussi de concevoir ce développement comme celui de l'ensemble de la société et pas comme une simple croissance économique.

Pour la Charte de Développement, la crise actuelle est avant tout culturelle et le premier objectif à atteindre doit être de favoriser l'émergence d'une identité commune qui prenne en compte les différences culturelles des communautés présentes à Tahiti et dans les archipels.

Moins catégorique, le rapport du CESC parle de crise multiforme, à la fois économique, politique, culturelle et existentielle.

Les deux documents s'accordent pour souligner que la culture d'un peuple, c'est sa manière d'être, d'avoir, de se tenir et de s'exprimer sur le plan économique, politique et social. Le problème crucial est alors d'harmoniser les apports de la culture autochtone et ceux d'un système importé privilégiant le marché. 
La croissance économique, vecteur privilégié de cette nouvelle identité

Les doléances exprimées par la Charte de Développement ont été entendues plus que prises en considération et le Pacte de Progrès reflète avant tout le point de vue de gestionnaires qui font prévaloir l'ordre établi et l'équilibre des grands agrégats sur les réformes structurelles et les choix de société. Les mesures qu'il préconise se cantonnent dans le champ de l'économie et sont disjointes des questions sociales qui ne sont abordées que de façon subsidiaire, sous un éclairage technique, comme si une reprise de la croissance économique allait résoudre les problèmes pendants. Inversant les priorités définies par les instances de réflexion dans la Charte de Développement, le Pacte insiste sur la nécessité de compenser dans les meilleurs délais le manque à gagner découlant du départ du CEP. Prenant ses distances vis-à-vis de réflexions de caractère existentiel sur les problèmes de société et d'identité, il marque le retour à des préoccupations plus terre à terre d'ordre économique et financier.

Après s'être auto-saisi et avoir souligné que les enjeux n'étaient pas seulement économiques mais culturels, sociaux et politiques, le CESC revient lui-même à sa fonction première qui n'est que consultative et se borne à donner des avis au gouvernement en tant que représentant de la société civile et des forces vives du Territoire.

En 1995, en prévision de l'arrêt définitif des essais et, cette fois, à la demande expresse du président du gouvernement, il se penche à nouveau sur l'avenir du Territoire et fait des propositions sur les infléchissements à apporter au Pacte de Progrès dans l'optique d'un développement durable qui ne s'appuie plus sur la rente nucléaire.

Le CESC commence par prendre ses distances avec ceux qui pensent que "seul un retour aux valeurs traditionnelles du passé pourra sortir la Polynésie française de la spirale infernale du tout économique qui caractérise la société moderne » et se range du côté des "plus nombreux qui estiment que le choix imposé de la modernité et les habitudes passées sont irréversibles et qu'il faut continuer dans cette voie, tout en corrigeant les erreurs du passé et en centrant le développement autour et au profit de l'homme ».

Il prend aussi le contre-pied de ceux qui pensent que la crise actuelle est liée à la perte d'identité des Polynésiens de souche. Une autre lecture des résultats du recensement de 1988 l'amène à constater que plus de $87 \%$ de la population est constituée de 'Polynésiens de souche et apparentés'. Il en déduit que les problèmes rencontrés sont moins liés à des questions d'identité culturelle qu'à une situation sociale qui ne diffère pas significativement de celle qu'on peut rencontrer dans les banlieues des grandes villes, un peu partout dans le monde.

Dans la mesure où la culture n'est pas un ensemble figé, exalter un passé mythique lui semble une « perversion du culturel ». Et, plutôt que de se référer à une société maohi révolue, qui était moins paisible et harmonieuse qu'on ne veut bien le croire, mieux vaut s'appuyer sur la Polynésie d'aujourd'hui, qui résulte de la fusion des cultures maohi, occidentale et chinoise, pour construire une nouvelle identité polynésienne fondée sur la libre adhésion d'individus, indépendamment de leur origine ethnique, de leur religion et de leur couleur de peau. Ainsi pourra se construire " une nouvelle identité polynésienne composée d'hommes et de femmes rassemblés dans un creuset commun (la Polynésie), ayant un même objectif (construire un pays) et une même volonté (vivre ensemble un destin partagé) ».

Face à une économie de comptoir fondée sur une rente nucléaire qui n'a profité qu'à une minorité, face à des dirigeants obnubilés par les jeux du pouvoir et face à la gabegie des fonds publics par une administration pléthorique, c'est à la société civile qu'il appartient de corriger ces travers et de donner à la société un nouvel élan. Il faut pour cela réduire la part du secteur public, favoriser les initiatives du secteur privé et se tourner vers des activités d'exportation. Plutôt que d'essayer vainement de mettre en place une " société pseudo égalitaire », il faut la « tirer vers le haut » en tempérant les excès du libéralisme par des mesures de solidarité compensatoires: « Le Polynésien d'aujourd'hui et celui de demain doit être compris comme un individu qui choisit librement de vivre en Polynésie, quelles que soient son origine, son ethnie, sa religion. Il adhère ainsi à une entité polynésienne plurielle faite de respect mutuel, de tolérance, de solidarité et de liberté individuelle ».

Le même point de vue se retrouve dans un autre rapport sur l'après CEP élaboré par un parlementaire polynésien qui formule des observations du même ordre sur la nécessaire réduction des coûts de production et la limitation des avantages de la fonction publique. En matière de formation, il conseille d'éviter ce qu'il qualifie de « complexe de l'ethnologue » et de « dérive culturelle ». Donnant la priorité à l'instruction sur l'éducation culturelle, il suggère de placer les jeunes dans « la perspective du $\mathrm{XXI}^{\mathrm{e}}$ siècle et non pas à l'époque - obscure - des grandes migrations ». 
Face à une crise multiforme qui ne peut qu'être aggravée par la disparition des transferts liés au CEP, l'option retenue doit, en définitive, parer au plus pressé et soutenir l'effort financier de l'État en le subordonnant à la mise en œuvre d'une politique contractuelle de développement. Elle doit permettre de répondre aux problèmes $\mathrm{du}$ moment et lève provisoirement l'hypothèque qui a conduit à la crise d'identité des années passées. Elle ne résout pas pour autant les problèmes de dépendance qui en sont, pour une large part, à l'origine.

\section{La construction d'une nouvelle identité pour sortir de la crise}

En août 1996, une nouvelle convention entre l'État et la Polynésie française vient renforcer l'autonomie économique du Territoire. L'État s'engage à maintenir pendant dix ans le niveau des flux financiers résultant de l'activité du CEP et la Polynésie s'engage à mettre en œuvre un « programme stratégique » lui permettant « d'assurer sa reconversion globale dans les domaines économique, social et culturel, avec comme objectif de remplacer les activités liées à l'existence du CEP par des activités appuyées sur ses ressources propres $»$.

Ce Programme Stratégique, qui s'inscrit dans le prolongement du Pacte de Progrès, est financé par un Fonds pour la reconversion économique placé sous le double contrôle de l'État et du Territoire. Il doit permettre à ce dernier de reconstruire son économie "dans le cadre d'un processus de développement équilibré qui cimente la cohésion sociale et préserve les valeurs culturelles de la Polynésie ». La priorité est donnée au développement des secteurs productifs mais d'autres mesures d'accompagnement sont également prévues.

Sur le plan culturel, elles s'attachent, comme le Pacte de Progrès, à forger " une identité culturelle plurielle et pluriethnique qui doit constituer le ciment de la société polynésienne et permettre à chacun de trouver sa place ». C'est au gouvernement qu'il appartient de faciliter l'émergence de cette identité nouvelle en donnant à chacun la possibilité de s'enrichir au contact de l'autre en soutenant la culture traditionnelle aussi bien que la culture moderne.

Avec un optimisme qui contraste avec l'inquiétude exprimée par la Charte de Développement, quinze ans plus tôt, le texte de présentation du Programme stratégique estime que "cette démarche basée sur une synthèse harmonieuse pourra rassembler les Polynésiens autour de valeurs telles que la convivialité, le respect de la vie, le goût de la beauté de l'équilibre et de l'harmonie naturelles, le sens de la fête. Ces valeurs sont et doivent demeurer cet art de vivre polynésien unique, qui constituera le cadre indispensable à la cohésion et à l'unité du peuple polynésien à l'entrée du XxI' siècle » (Journal officiel de la Polynésie française du 21 août 1997).

L'autonomie économique que poursuit le Programme stratégique doit venir compléter l'autonomie politique que renforce le statut de juillet 1996. L'une et l'autre doivent se renforcer mutuellement et donner au Territoire une identité qui ne se confonde pas avec celle de l'État. Cette redéfinition des rapports entre l'État et le Territoire prend aussi appui sur la volonté de Paris d'établir avec l'outre-mer des relations moins asymétriques, davantage basées sur le partenariat que sur l'assistance.

\section{Passage d'un régime de tutelle à un régime plus autonome}

La difficulté est que l'assouplissement de la tutelle de Paris substitue à son hégémonie celle de Tahiti. Il ne prend pas suffisamment en compte le fait que la Polynésie, loin de se limiter aux îles de la Société, englobe quatre archipels satellites répartis sur 4 millions de $\mathrm{km} 2$.

Depuis la fin des années 1970, les réformes statutaires qui se sont succédées ont conféré au Territoire une autonomie croissante qui a été qualifiée d'" autonomie de gestion » en 1977, d'« autonomie interne » en 1984 et d'« autonomie renforcée » en 1996.

La réforme de 1977 (loi $\mathrm{n}^{\circ} 77-772$ du 12 juillet 1977), qui s'inscrit dans une logique de décentralisation plus que d'autonomie, débouche, pour la première fois, sur un véritable gouvernement territorial doté d'une large marge de manœuvre en matière de gestion. La contribution de la métropole au budget du Territoire n'est plus globale mais remplacée par des aides spécifiques négociées de ministère à ministère et orientées vers des objectifs déterminés. L'État ne se départit pas, pour autant, de ses moyens de contrôle.

Le statut de 1984 (loi n ${ }^{\circ} 84-820$ du 6 septembre 1984) est le premier à reconnaître l'identité polynésienne et les symboles qui s'y rattachent comme un drapeau, un hymne et un sceau territoriaux. Le Territoire jouit officiellement d'une autonomie interne mais ni le haut-commissaire, représentant l'État, ni le président du gouvernement territorial, ne se voient reconnaître la qualité de chef du territoire. Le sénateur Daniel Millaud justifie cette ambiguïté par le fait qu'en tahitien les termes d'autonomie et d'indépen- 
dance s'expriment par le même terme tiamaraa et que c'est le qualificatif « interne » qui permet d'en préciser le sens.

Le statut de 1984, comme les précédents, s'apparente néanmoins davantage à la décentralisation mise en œuvre en métropole qu'à l'autonomie dont il se réclame. Il accroît à nouveau la latitude du territoire en matière de gestion et consacre l'ascension d'une élite dirigeante locale qui contrôle désormais des rouages clés du pouvoir.

Le statut d'autonomie de 1996 (lois organiques $\mathrm{n}^{\text {os }}$ 96-312 et 96-313 du 12 avril 1996) se traduit à nouveau par des accroissements ponctuels de la marge de manœuvre des autorités territoriales et son côté le plus novateur est de remplacer dans la dénomination officielle des institutions territoriales le terme de territoire par celui de Polynésie française. Il précise également que la République garantit son autonomie et "favorise l'évolution de cette autonomie de manière à conduire ce territoire d'outre-mer au développement économique, social et culturel, dans le respect de ses intérêts propres, de ses spécificités géographiques et de son identité » (article 1).

Le terme d'autonomie n'en reste pas moins ambigu et fait l'objet d'interprétations diverses. Dans le partage des compétences, les élus polynésiens tendent en effet à se référer au système anglo-saxon de " self government », qui prévaut dans le Pacifique, et les représentants de l'État restent attachés à la notion d'un État unitaire s'exerçant dans le cadre de la République.

$\mathrm{Si}$ on passe du plan théorique au plan pratique, on peut constater que les rapports du Territoire avec l'État se trouvent redéfinis dans le cadre des nouveaux statuts, mais qu'il n'en va pas de même des rapports entre l'administration centrale et l'administration territoriale. Les services centraux parisiens restent en effet mal informés et souvent déroutés par des réalités polynésiennes qui diffèrent de celles d'autres collectivités territoriales, métropolitaines ou ultramarines.

\section{Infléchissement d'une politique d'assistance vers une politique de partenariat}

Le passage d'un régime de tutelle à un régime d'autonomie renforcée doit logiquement avoir pour corollaire le passage de relations d'assistance à des relations de partenariat. Dès 1992, le CESC souligne que le développement du Territoire ne se réduit pas à l'alternative entre une croissance tributaire de la métropole et un développement autocentré s'appuyant sur les res- sources du crû, et déclare qu'il «ne peut être le fruit que d'un partenariat entre l'ensemble des parties en présence $»$.

Le Pacte de Progrès et le Programme stratégique, à la différence des Plans d'Ajustement Structurel (PAS) mis en œuvre par la Banque mondiale et le FMI, se caractérisent ainsi par le fait que l'aide n'est apportée qu'à des plans à long terme élaborés par les intéressés eux-mêmes sur la base d'un large consensus. Ces plans se situent dans l'esprit des contrats de développement préconisés par l'OCDE et l'Union européenne et ajoutent à l'objectif de croissance celui d'une distribution plus équitable de ses bénéfices.

Ils définissent des objectifs à cinq ans dans le cadre de contrats de développement de même durée. C'est le cas du Pacte de Progrès qui court de 1994 à 1999 et du Programme stratégique qui va de 2000 à 2005. Ces contrats, longuement négociés, ne sont pas contraignants mais seulement incitatifs et leur mise en œuvre implique une étroite collaboration entre les acteurs institutionnels et leurs partenaires publics ou privés.

Il reste que l'État a du mal à se départir d'une tradition jacobine et centralisatrice et les scandales financiers, qui ont récemment émaillé l'actualité du Territoire, ne peuvent que le conforter dans une attitude calquée sur le principe que celui qui paie contrôle.

Le rapport du Commissariat général au Plan sur l'outre-mer de 1993 laisse entendre que ces dérives financières sont liées à une " autonomie qui exclut tout contrôle». Il conseille, en conséquence, à l'État de mettre en œuvre une politique très volontariste pour faire prévaloir « un scénario de ressaisissement » sur un "scénario au fil de l'eau » qui risque de se transformer en «scénario catastrophe $\gg$.

Force est de constater que le Pacte de Progrès et le Programme stratégique, initiés au cours de cette période, sont mis en œuvre par un pouvoir centralisé interventionniste qui continue de s'appuyer sur les institutions plus que sur le marché, et sur les agents de l'État plus que sur les citoyens.

\section{Limites et ambiguïtés de la quête identitaire du Territoire}

Ce qui était vrai hier de l'État l'est aujourd'hui du Territoire et l'hégémonie de Tahiti n'a cessé de se renforcer. Elle se manifeste par la place éminente qu'occupe la capitale dans les rouages territoriaux, aussi bien que par les faibles résultats de la politique de revitalisation des archipels éloignés amorcée en 1975. Elle est renforcée par le passage, au milieu des années 1980, d'un déve- 
loppement autocentré fondé sur une politique de substitution d'importations à un développement d'orientation libérale tourné vers l'extérieur qui privilégie les exportations. Y correspond le passage d'une stratégie « bottom up » à une stratégie « top down » qui, plutôt que de résoudre les problèmes par le bas, essaie de le faire par le haut et envisage de passer directement d'une économie de subsistance à une économie de services basée sur des prestations "haut de gamme». Alors que les îles éloignées restent à l'écart, mal reliées entre elles et mal équipées, le pôle urbain de Papeete se renforce et se présente comme un symbole de modernité en prise directe sur le monde extérieur et mieux connecté avec la métropole, les États-Unis ou Taiwan qu'avec ses îles périphériques. En sens inverse, certains archipels éloignés qui possèdent une langue, une culture et une histoire distinctes de celles de Tahiti, sont tentés de tourner leurs regards vers le Japon, les États-Unis ou la métropole plutôt que vers Tahiti qui les néglige et ne tient pas assez compte de leurs spécificités (Blanchet, 1998).

Comme le soulignent une fois de plus les sénateurs Lucien Lanier et Guy Allouche au terme d'une mission effectuée en Polynésie française en janvier 1996, «le développement du Territoire s'est jusqu'à présent effectué sur un mode centralisateur, privilégiant les îles de la Société et laissant à l'écart les autres archipels [...]. La diversité géographique et culturelle est largement passée sous silence, de même que l'organisation des communes et les compétences qui leur sont dévolues ».

Dans une déclaration annexée au rapport sur l'après-CEP, l'un des vice-présidents du CESC fait état $\mathrm{du}$ "nouvel impérialisme » qui règne dans les communes où le « tout État » d'avant l'autonomie interne a été remplacé par le « tout Territoire $»$.

Le propos est peut-être excessif mais force est de constater que le statut de 1996, comme ceux qui le précèdent, se concentre sur le partage des compétences entre l'État et le Territoire et, au sein du Territoire, sur la répartition des pouvoirs entre le gouvernement et l'Assemblée territoriale, tous deux basés à Tahiti. Il comporte peu de dispositions relatives aux institutions locales que constituent les communes. Il précise simplement que l'État et le Territoire leur apportent leur concours dans les domaines qui leur sont dévolus et rappelle la compétence de l'État en matière d'administration communale. Depuis la réforme de 1971, les communes de Polynésie française forment en effet des collectivités territoriales de la République de droit commun, et non de droit local, et sont directement gérées par
l'État. Un des objectifs de la réforme était de mieux répartir les fonds publics et de mettre fin à une discrimination budgétaire qui s'exerçait au détriment des archipels éloignés. Il permettait, en même temps, à l'État de s'appuyer sur ces communes périphériques pour faire pièce au gouvernement territorial, partisan d'une autonomie interne qu'il n'était alors pas question d'encourager.

Les communes sont toujours l'enjeu d'une lutte de pouvoir entre le Territoire, qui conteste la mainmise de l'État sur ce qu'il continue d'appeler les «communes de l'État», et l'État qui se refuse à y étendre une décentralisation qui se ferait au bénéfice de Tahiti. Ce sont finalement les communes qui pâtissent de cette situation et continuent d'être "sacrifiées sur l'autel des contraintes budgétaires » (Al Wardi, 1998).

Cette situation est d'autant plus regrettable qu'avec la poussée démographique et le mouvement de retour dans les îles comme avec les travaux d'infrastructure réalisés dans le cadre de la politique de revitalisation des archipels, les communes doivent faire face à des charges croissantes sans avoir les moyens d'y faire face. Leurs ressources proviennent avant tout des transferts en provenance de l'État et elles ne peuvent ellesmêmes lever de recettes fiscales, faute de moyens et aussi parce qu'il s'agit là d'un domaine de la compétence exclusive du Territoire. Une loi de juillet 1990 a fait un pas en direction des communes en instituant dans chaque archipel un Conseil, composé des membres de l'Assemblée territoriale et des maires de la circonscription. Ce Conseil d'archipel doit être obligatoirement consulté par le Président du gouvernement sur les plans de développement, les contrats de plan comme sur les dessertes maritimes et aériennes. Il doit aussi avoir la possibilité de donner son avis dans tous les domaines qui le concernent. Mais l'Assemblée territoriale n'a jamais délibéré sur l'organisation et le fonctionnement de ces Conseils si bien qu'ils n'ont pas encore vu le jour.

Pour désenclaver et favoriser le développement des archipels éloignés, le Programme stratégique prévoit aussi que les communes pourront bénéficier $\mathrm{du}$ fonds pour la reconversion économique. Mais, soucieux de contrôler l'emploi de ces fonds, le gouvernement territorial s'empresse de créer une délégation pour le développement des communes qui doit lui permettre d'intervenir en fonction des priorités qu'il aura lui-même retenues. Quelques mois plus tard après l'adoption de ces mesures, à l'instigation de l'État, un addendum au Programme stratégique est venu rappeler que les communes doivent être considérées comme partenaires de la recon- 
version économique et qu'il faut privilégier une démarche de concertation. Une majorité de communes se disent en effet favorables au maintien d'un régime de contrôle a priori de l'État, ce qui peut s'expliquer par plusieurs raisons. Sur le plan historique, au moment de leur création, elles ont été calquées sur les anciennes chefferies et le maire a pris la suite du chef de district qui avait lui-même remplacé le chef local qui appartenait à la classe noble des arii. Aujourd'hui encore, dans les îles éloignées, le rôle et la fonction de maire dépassent largement ceux d'un simple élu. La plupart d'entre eux sont confrontés de façon chronique à une situation financière délicate qui les fait dépendre de l'extérieur pour leurs dépenses comme pour leurs recettes. Cette situation tend à redonner vie à une longue tradition de clientélisme et les soumet aux aléas de la politique politicienne. Elle renforce paradoxalement leur dépendance de Tahiti au moment où Tahiti commence à se libérer de l'emprise de l'État.

\section{Conclusion}

En guise de conclusion, on constate qu'en Polynésie française, comme ailleurs, c'est en période de crise que l'identité ne va pas de soi et qu'on s'interroge sur elle avec un mélange d'inquiétude et d'espoir. Les lectures qui en sont proposées, sur un plan général comme sur le plan institutionnel, montrent qu'il est difficile d'en isoler les composantes culturelles des composantes à caractère politique, économique ou social. Elles montrent aussi que, loin d'être primordiale et immuable, l'identité se présente, au contraire, comme un assemblage et une construction provisoires qui n'excluent pas les ambiguïtés, les contradictions et les conflits. Elle fait l'objet de stratégies variées où voisinent l'appétit de pouvoir d'élites nouvelles en mal d'intégration, la fierté compensatoire de populations tenues à l'écart ou encore le glissement d'hégémonie qui s'opère à la faveur de l'affaiblissement de l'État-nation et d'un déplacement de ses centres d'intérêt.
Dans la mesure où elle fait appel aux ressources de l'imaginaire, à de nouveaux métissages et à une synthèse originale de l'ancien et du moderne, elle peut aussi se révéler porteuse d'avenir et source d'avantages comparatifs face à un État dont on n'attend plus qu'il résolve tous les problèmes.

\section{RÉFÉRENCES}

Al WARDI Sémir, 1998. Tahiti et la France. Le partage du pouvoir, Paris, l'Harmattan, $312 \mathrm{p}$.

Blanchet Gilles, 1994. La Polynésie française à la croisée des chemins, Mondes en développement, tome 22, 86, Louvain, pp. 23-36.

—, 1998. Expressions et constructions identitaires aux îles Marquises. Conférence donnée à l'École des Hautes Études en Sciences Sociales (EHESS) dans le cadre du GDR «Identités et transformations des sociétés océaniennes », Paris, janvier 1998, 7 p.

Comité ÉCONOMique, SOCIAL ET CULTURel (CESC), 1992. Quels choix pour l'avenir de la Polynésie?, Papeete, avis $\mathrm{n}^{\circ} 82,87 \mathrm{p}$.

—, 1995. Quel avenir pour le Territoire après l'arrêt des essais? Papeete, avis $n^{\circ} 98,78 \mathrm{p}$.

Commissariat géNÉral du Plan, 1993. Outre-Mer : le défi des singularités. Préparation du $\mathrm{XI}^{\mathrm{e}}$ Plan. Paris, La Documentation française, $214 \mathrm{p}$.

LANier Lucien et Guy AlLouche, 1996. La Polynésie après l'arrêt des essais nucléaires. L'autonomie institutionnelle au service de développement économique. Rapport du Sénat n ${ }^{\circ} 215 ., 45$ p.

Pacte de Progrès économique, SOCIAL ET CULturel DE La Polynésie franÇaISE, 1993. Papeete, éditions Charte du Développement [1 ${ }^{\text {ère }}$ partie : propositions de la délégation polynésienne; $2^{\text {ème }}$ partie : principales références pour l'élaboration du Pacte de Progrès].

RAOULT Éric (rapporteur), 1994. Rapport sur le projet de loi définissant les orientations de l'État en faveur du développement économique, social et culturel du territoire de la Polynésie française, Assemblée nationale, $\mathrm{n}^{\circ} 929,45 \mathrm{p}$. 